\section{Management of cancer-related pain}

In the article by Cipta and colleagues ${ }^{1}$ reviewing cancerrelated pain management, after discussing cancer pain syndromes and their many sources, they turn their attention to the assessment and management of those cancer-related pains. However, there is no mention of breakthrough pain in cancer patients (BTCP) either in regard to assessment or management, including the use of transmucosal fentanyl for its treatment.

Their discussion of pain assessment highlights the need to obtain a detailed pain history from the patient that includes intensity, quality, and aggravating factors of the cancer-related pain. It is also necessary to establish the history of the temporal features of the pain (that is, is it constant or episodic?) as well as the patient's response to prior and current regimens for the management of pain. This information is key in the diagnosis of breakthrough pain in this oncology population. As Caraceni and colleagues have noted, recognition of this "separate and characteristically different presentation that is breakthrough pain" is crucial in assuring that it is adequately managed. ${ }^{2}$

Breakthrough pain has been defined in guidelines as "transitory exacerbations of pain that occur on a background of stable pain otherwise adequately controlled by aroundthe-clock opioid therapy"3 or as "a transient exacerbation of pain that occurs either spontaneously, or in relation to a specific predictable or unpredictable trigger, despite relatively stable and adequately controlled background pain." ${ }^{\prime \prime}$ These definitions reinforce the importance of taking a detailed pain history, as noted, and the review of prior and current treatments. Given the known barriers to effective pain management in patients with cancer, knowledge of breakthrough pain is important in the reduction of some of those barriers.

National Comprehensive Cancer Network guidelines ${ }^{5}$ specifically address breakthrough pain as "pain that fails to be controlled or 'breaks through' a regimen of regularly scheduled opioid." The guideline, which is pending a revised and updated release, further indicates a categorization of breakthrough pain. As such, categorization can have direct impact on treatment. Breakthrough pain can be classified as either spontaneous or idiopathic pain because the episodes are not related to an identifiable precipitant, and so are unpredictable in nature; or categorized as incident or precipitated pain because the episodes are related to an identifiable event or action and are therefore predictable in nature. ${ }^{4}$ In addition, there is the potential for further classification of incident pain as volitional (eg, due to a volitional act such as walking), nonvolitional (eg, due to a nonvolitional act such as coughing), or procedural (eg, due to a therapeutic intervention such as radiation therapy or wound dressing). However, background pain that is uncontrolled or poorly controlled near the end of a pain medication dose should not be categorized as break- through pain. ${ }^{2}$ Pain as a result of the decreasing concentration of analgesic should not considered as breakthrough pain, but rather referred to as "end-of-dose failure."

Breakthrough pain typically has a rapid onset, taking just a few minutes to reach peak pain, is of short duration (generally less than an hour), and is of high severity, with the number of daily episodes sometimes surpassing $10 .{ }^{2}$ Patients who experience breakthrough pain have significantly greater pain-related functional impairment, psychological distress, and a significantly reduced quality of life. ${ }^{2}$ The recognition of breakthrough pain as such is key to its proper management and resulting improvement in the patient's life.

A number of treatments are discussed in Cipta and colleagues, ${ }^{1}$ including both immediate and controlled-release opioids. Although fentanyl is mentioned in a long-acting transdermal formulation and by not having active metabolites, making it a safer alternative in patients with underlying renal disease as other opioids have active metabolites that may be neurotoxic under such circumstances; it is not mentioned in its relevant form: transmucosal immediate-release fentanyl (TIRF). The US Food and Drug Administration-approved TIRFs, which have been available since the 1990s, are specifically indicated for the management of breakthrough pain in cancer patients aged 18 years or older who are already receiving and are tolerant to opioid therapy for their underlying persistent cancer pain.

In a review of the 6 rapid-onset formulations of fentanyl approved in the United States for the management of BTCP, the different fentanyl formulations may vary in pharmacokinetic properties and ease of use, but all have a rapid onset and a relatively short duration of analgesia. ${ }^{6}$ In particular, fentanyl sublingual spray was reviewed and indicated to have absorption within 5 minutes of administration, plasma concentrations increasing over the first 30 minutes and remaining elevated for 60-90 minutes (pharmacokinetic studies in healthy subjects). ${ }^{6}$ Given the rapid rise in the pain intensity and limited duration of the episodes of BTCP, TIRFs should be considered in the indicated patient population for treatment of BTCP when pain cannot be attributed to inadequate dosing of their aroundthe-clock opioid. ${ }^{5}$

As uncontrolled pain is both feared and debilitating for cancer patients, ${ }^{1}$ the recognition and proper management of BTCP will assist in the goal of the relief of the burden of cancer patients in tolerating their journey with their disease and optimizing their quality of life. ${ }^{1}$

\section{Lewis M Fredane, MD (lfredane@insysrx.com) Chandler, Arizona}

\section{Disclosures}

Dr Fredane is vice-president of medical affairs at Insys Therapeutics, manufacturer and distributor of SUBSYS (fentanyl sublingual spray). 


\section{References}

1. Cipta AM, Pietras CJ, Weiss TE, MD, Strouse TB. Cancer-related pain management in clinical oncology. J Community Support Oncol. 2015;13:347-355.

2. Caraceni A, Davies A, Poulain P, Cortés-Funes H, Panchal SJ, Fanelli G. Guidelines for the management of breakthrough pain in patients with cancer. J Natl Compr Canc Netw. 2013;11(suppl 1):S29-36.

3. Caraceni A, Hanks G, Kaasa S, et al. Use of opioid analgesics in the treatment of cancer pain: evidence-based recommendations from the EAPC. Lancet Oncol. 2012;13:e58-68.

Authors' response - Dr Fredane's input is appreciated and valuable, both his discussion of breakthrough pain in cancer patients, and that it is important to consider all available rapid-onset analgesics for optimal management of cancer-related breakthrough pain. As explained in Dr Fredane's letter, TIRF may be of particular benefit for patients aged 18 years or older who are already receiving and have become tolerant to opioid therapy. Fentanyl does not accumulate or lead to increased side effects in the setting of renal failure, and is thus a preferred opioid in advanced kidney disease.

Transmucosal formulations are absorbed through the buccal
4. Davies AN, Dickman A, Reid C, et al. The management of cancer related breakthrough pain: recommendations of a task group of the Science Committee of the Association for Palliative Medicine of Great Britain and Ireland. Eur J Pain. 2009;13:331-338.

5. Swarm RA, Abernethy AP, Anghelescu DL, et al; National Comprehensive Cancer Network. Adult cancer pain. J Natl Compr Canc Netw. 2013;11:992-1022.

6. Taylor DR. Single-dose fentanyl sublingual spray for breakthrough cancer pain. Clin Pharmacol. 2013;5:131-141.

mucosa, thereby avoiding first-pass metabolism in the liver by direct venous uptake and drainage into the systemic circulation. This may provide an additional benefit when compared with oral routes, because transmucosal formulations can result in higher bioavailability and faster onset of action. This pharmacologic characteristic can be particularly useful in breakthrough pain, which can often be rapid and transient in its presentation.

Our review article was incomplete without mention of breakthrough pain and the transmucosal fentanyl products. We appreciate the correction. 